\title{
Lavoro, consumo e tempo libero nelle pratiche informali di riciclo alimentare. Il reciclaje nella città di Granada (Spagna)
}

Work, consumption and free time in food-recycling's informal practices. The reciclaje in the city of Granada (Spain)

\section{Giorgio Cassone}

\section{(2) OpenEdition Journals}

Edizione digitale

URL: http://journals.openedition.org/aam/1572

DOI: $10.4000 /$ aam. 1572

ISSN: 2038-3215

\section{Editore}

Dipartimento Culture e Società - Università di Palermo

\section{Notizia bibliografica digitale}

Giorgio Cassone, «Lavoro, consumo e tempo libero nelle pratiche informali di riciclo alimentare. II

reciclaje nella città di Granada (Spagna) », Archivio antropologico mediterraneo [Online], Anno XXII, n. 21

(1) | 2019, online dal 30 juin 2019, consultato il 23 septembre 2019. URL : http://

journals.openedition.org/aam/1572; DOI : 10.4000/aam.1572

Questo documento è stato generato automaticamente il 23 septembre 2019

Archivio antropologico mediterraneo 


\title{
Lavoro, consumo e tempo libero nelle pratiche informali di riciclo alimentare. Il reciclaje nella città di Granada (Spagna)
}

\author{
Work, consumption and free time in food-recycling's informal practices. The \\ reciclaje in the city of Granada (Spain)
}

\section{Giorgio Cassone}

Siamo portati a ritenere poveri cacciatori e raccoglitori

perché non hanno nulla; ma forse proprio per questo

dovremmo ritenerli liberi.

(Sahlins 1980: 25-26)

1 Nel 2006 vinsi una borsa per un soggiorno di studio presso l'università di Granada ed ebbi così la possibilità di vivere un anno nel contesto estremamente multiculturale della cittadina andalusa. M'immersi nell'animata vita granadina cogliendo le svariate occasioni di socialità che la città offriva e fui profondamente arricchito dalla scoperta di modi di vita e di pratiche fino ad allora a me sconosciuti o da me stigmatizzati. Una di queste pratiche è ciò che localmente viene indicato con il termine "reciclaje" . Questo termine indica la ricerca, il recupero, la distribuzione e il consumo di cibo scartato dal ciclo di distribuzione alimentare urbano, e la trasformazione di questi scarti in cibo consumato individualmente o collettivamente, dunque in un oggetto (nuovamente) commestibile e al tempo stesso economico e sociale.

Conobbi il reciclaje facendomi includere, quasi senza rendermene conto, nei circuiti di condivisione e di reciprocità che il reciclaje stesso crea. In effetti, durante quell'anno da borsista mi sono regolarmente nutrito con gli alimenti recuperati nei cassoni del mercato all'ingrosso della città, che ogni settimana un'amica donava a me e alla mia coinquilina. Tuttavia, è stato solo durante una ricerca etnografica condotta nel 2009 
che ho potuto cominciare a osservare nel dettaglio e a partecipare personalmente a questa pratica informale di recupero di cibo invenduto e scartato dai centri di distribuzione. Negli anni seguenti, nell'ambito del mio percorso accademico, ho realizzato più ricerche etnografiche sulle pratiche di riciclo alimentare in diversi terreni, in Spagna e Francia².

3 Partendo dal mio terreno etnografico nella città granadina ${ }^{3}$, in quest'articolo descriverò il riciclo alimentare ipotizzando che esso costituisca un'attività lavorativa legata alla ricerca di una parte dei beni necessari alla sussistenza di coloro che la praticano; mostrerò come nasca e viva ai margini del sistema di produzione dominante - e parallelamente a esso - e come sia funzionale alla pratica di uno stile di vita "alternativo". Successivamente tenterò di presentare i prerequisiti del reciclaje utilizzando i concetti antropologici di lavoro, consumo e tempo libero. Mostrerò come la rinuncia al consumo (nel senso di consumismo) e a un lavoro convenzionale risultino i fattori principali che favoriscono la pratica del reciclaje. Infine, analizzerò le conseguenze economico-sociali della pratica del reciclaje: esse consistono soprattutto nella creazione in un nuovo ciclo di vita "sociale" (Kopytoff 1986) degli alimenti riciclati. Il cibo scartato, nella sua (ri)messa in circolazione, permette di creare reti di reciprocità in cui la scoria del sistema si trasforma in una nuova produzione. Attraverso un particolare ciclo di distribuzione, essa viene poi consumata individualmente o collettivamente. In altre parole, grazie al lavoro di riciclo, gli alimenti passano da uno stato di scoria dell'attività di produzione a una nuova vita, acquistano un valore d'uso $\mathrm{e}$ di scambio che prima non avevano. Così facendo questi nuovi oggetti economici divengono l'elemento funzionale al mantenimento di uno status sociale alternativo ai modelli dominanti di consumo e alla creazione di reti di solidarietà e condivisione (Orlando 2018) che sfociano in un'economia parallela a quella di mercato. Un'economia creata da individui appartenenti a quella che Bauman definisce una sorta di "sottoclasse" costituita da "consumatori difettosi" (2009: 152-154).

\section{Spigolature}

4 Il termine reciclaje indica la ricerca assidua e minuziosa di cibo scartato dal ciclo di produzione e distribuzione, e depositato nei cassonetti dell'immondizia urbana. Il cibo recuperato è successivamente destinato al consumo umano allo stato in cui si trova o a seguito di una successiva lavorazione. Agnes Varda $(2000,2002)$ segnala un legame tra questa pratica urbana e la spigolatura contadina ${ }^{4}$. Tuttavia ciò che differenzia maggiormente la spigolatura contadina dal reciclaje è che la prima concerne una "materia prima" (il grano o delle produzioni agricole più in generale) mentre l'altra investe una "materia ultima" (i rifiuti).

5 L'elenco degli alimenti che si riciclano è molto lungo. Nei contesti come quello spagnolo, osservato negli anni di ricerca di terreno, il mercato degli alimenti offre in ogni stagione frutta e verdura di ogni tipo, proponendo la stessa varietà anche a coloro che frugano nelle sue immondizie. Nei mesi invernali della mia ricerca era molto probabile trovare dei pomodori che sono un tipico prodotto estivo. Accadeva lo stesso con zucchine, melanzane, peperoni, cetrioli, avocado, meloni estivi, angurie, ecc. Trovare questi alimenti fuori stagione in inverno nei rifiuti era facile come trovare quelli di stagione: porri, broccoli, cavoli, melagrane, agrumi in generale. 


\section{Granada}

In una città di 300.000 abitanti come Granada ${ }^{5}$, i luoghi dove praticare il reciclaje sono numerosi e disseminati sul territorio urbano. Si può praticare il reciclaje nel retro di un supermercato all'ora di chiusura, lungo i viali del mercato settimanale, nel cuarto basura $^{6}$ di un piccolo mercato coperto o, ancora, dal panettiere sotto casa alla fine della giornata. Ma il reciclaje non garantisce gli stessi risultati su ognuno di questi siti. È per questo che, per la riuscita di una buona giornata di reciclaje, è necessaria una buona conoscenza del territorio in cui s'intende recuperare alimenti.

7 Di questo mi accorsi attraverso la mia esperienza personale. All'inizio della ricerca passai numerose ore per più giorni consecutivi fuori dai supermercati, molto prima $\mathrm{e}$ molto dopo l'orario di chiusura, in attesa della comparsa dei riciclatori. Ogni attesa risultò vana. Nessun cassonetto dei rifiuti veniva portato all'esterno degli edifici, nessun cassonetto già presente, opportunamente da me controllato, risultava pieno, $\mathrm{e}$ ovviamente nessun riciclatore appariva all'orizzonte. Diversi tentativi falliti stavano riducendo radicalmente il mio ottimismo e la fede nella buona riuscita della ricerca. La causa di tutto questo era che non conoscevo i tempi e i luoghi del reciclaje. I punti d'osservazione erano stati scelti in modo casuale o sulla base di informazioni ricevute da persone in contatto con riciclatori, ma con una conoscenza solo superficiale del funzionamento del reciclaje. Scoprii i tempi e i luoghi del reciclaje quando incontrai i primi riciclatori. La prima a reindirizzare la mia ricerca fu Ilenia, una giovane studentessa di scienze politiche e niñera de noche ${ }^{7}$ che riciclava da anni. Fu lei a spiegarmi che a siti diversi corrispondono orari diversi, anche se è possibile riunirli in categorie specifiche.

\begin{tabular}{|c|c|c|}
\hline & Orari apertura attività commerciali & Orari reciclaje \\
\hline Supermercato & $9.15-21.15$ & $20.45-22.15$ \\
\hline Piccolo mercato & $7.00-14.50$ & $14.00-16.00$ \\
\hline Mercato Centrale & $5.00-12.00 / 13.00$ & $10.00-13.30$ \\
\hline Piccoli commerci & $8.00-14.00 / 17.00-20.30$ & $20.00-21.00$ \\
\hline
\end{tabular}

8 Benché sia generalmente diffusa l'idea che il reciclaje si realizzi solo alla fine del mercato, la tabella rivela che il momento del reciclaje si estende lungo l'ultima parte dell'attività lavorativa giacché la quantità di prodotti abbandonati aumenta col passare delle ore. Inoltre, si può notare che il reciclaje può essere svolto in due periodi distinti della giornata: dalle 10 alle 16 e dalle 20 alle 22.15. Questi due intervalli temporali corrispondono a luoghi diversi: nella prima parte della giornata si può riciclare nei mercati al dettaglio e nei mercati all'ingrosso nelle zone dedicate al deposito dei rifiuti, in serata il riciclo è possibile dopo la chiusura delle attività commerciali generalmente nei cassonetti posti nella parte posteriore degli esercizi o nelle vicinanze.

\section{Giovani riciclatori}

Durante la mia esperienza di campo ho notato che il reciclaje viene praticato da anziani e pensionati, persone senza fissa dimora, immigrati, gitani e giovani tra i 20 e i $35 \mathrm{anni}^{8}$. Quest'articolo si focalizza in particolare sull'analisi di quest'ultimo gruppo poiché i giovani riciclatori si distinguono dagli individui appartenenti agli altri gruppi per 
diversi aspetti. Nel contesto granadino, i giovani rappresentano la maggioranza dei riciclatori e, a differenza di altri recuperatori, sono presenti su tutti i siti di riciclo osservati (mercato centrale, mercato periferico, mercato all'ingrosso, supermercati).

Un altro aspetto che distingue i giovani riciclatori dai loro "omologhi" è il fatto che la pratica del reciclaje è descritta da costoro come la conseguenza di una volizione, un atto di volontà che riguarda la sfera del consumo. Ciò che li spinge ad agire è senza dubbio una necessità, indicata dai miei interlocutori come una necessità auto-indotta, frutto cioè di una loro consapevole scelta. Inoltre, mentre l'attività di riciclo praticata da pensionati, da persone senza fissa dimora, da immigrati e gitani si concentra soprattutto nella zona urbana, i giovani estendono le loro attività di riciclo anche fuori città, in prossimità del mercato all'ingrosso. Questo garantisce l'accesso a grandi quantità di cibo che successivamente vengono fatte circolare alimentando così le reti di vicinato e di interconoscenza, attraverso circuiti di condivisione e di reciprocità nei quali i giovani recuperatori sono inseriti.

11 L'approccio dei giovani alle immondizie è spudorato e noncurante dei giudizi altrui. Il solo disagio che mi è stato segnalato lo si prova la prima volta, quando le abitudini e il modo i cui sono considerate le immondizie si scontrano con l'esperienza diretta. Melissa, studentessa di logopedia e cameriera nei weekend, me lo spiegò durante un'intervista rispondendo alla mia domanda sul suo primo reciclaje:

Melissa: La prima volta m'imbarazzai un poco... in verità dicevo: "come? c'è da mettersi nelle immondizie? [...] mm, non so!"[...] e via così. Perché all'inizio ti da un poco di, cazzo, non so! Ti hanno sempre detto "non toccare le immondizie" e in quel momento... ti metti nelle immondizie, sai? Però alla fine vai, ti senti bene, sei contento, risparmi, e dici "buono". [...] E vai già con i pantaloni vecchi e gli stivali per metterti dentro! (Febbraio 2009. Traduzione dell'autore) ${ }^{9}$.

12 Superato questo primo blocco, sembra che non ve ne siano altri. Il riciclatore fruga, cerca con attenzione, esclude tutto ciò che non è commestibile o che non sarà più edibile nel giro di pochi giorni. La buona qualità e la grande quantità degli alimenti destinati al macero lo permettono. La sua ricerca è pacata e selettiva.

13 Generalmente il prodotto del lavoro del reciclaje non è nascosto o occultato. Il cibo viene riposto nei cartoni che vengono trovati nei rifiuti e con essi trasportato a casa. Questa particolare modalità di trasporto del cibo recuperato, visibile e facilmente disponibile, permette di trasformare un successivo incontro in un momento di scambio. Spesso, incontrando conoscenti lungo il cammino del ritorno a casa, i recuperatori donano una parte del loro lavoro di riciclo. Iaia, studentessa di Belle arti e venditrice ambulante di proprie produzioni artigianali, durante un'intervista mi ha detto: «Io se ce l'ho [il cibo], lo condivido e molte volte che vado a fare il reciclaje e ho già due buste e incontro uno che va a riciclare, gli lascio una busta». In questo modo per i beni alimentari recuperati non solo comincia un nuovo ciclo di vita economico-sociale ma essi vengono rimessi in circolazione, cioè inseriti in circuiti di conoscenza e reciprocità i quali fungono da mezzi di distribuzione in vista di un successivo consumo.

\section{Dopo il riciclo}

14 Terminata la raccolta degli alimenti nei mercati e nei supermercati, il cibo viene portato a casa. Ha inizio qui un'attività importantissima per l'ottimizzazione del lavoro di riciclo: la pulizia e la lavorazione degli alimenti. Quest'operazione, pur essendo 
semplice, richiede del tempo. Se per la pulizia del prodotto del reciclaje al piccolo mercato sono necessari al massimo trenta minuti, per la pulizia dei prodotti raccolti al mercato all'ingrosso e nei supermercati i tempi sono più lunghi. Dai report ${ }^{10}$ che seguono si può intuire la quantità di tempo necessaria a compiere l'intero processo di pulizia e trasformazione del cibo recuperato.

Tab. 1 - Report - Iaia - Mercato urbano

2 pomodori

2 arance

2 peperoni

1 avocado

1 cavolo

1 pera

1 lattuga

250 gr di funghi

$3 \mathrm{~kg}$ di cetrioli

Tab. 2 - Report - Melissa - Mercato ingrosso

$5 \mathrm{~kg}$ di pomodori

11 insalate "indivia"

6 lattughe

1 cavolo

6 limoni

10 peperoni verdi

6 peperoni rossi

3 mazzi di prezzemolo

1 mazzo di menta

1 mazzo di rosmarino

1 kiwi

10 zucchine

10 insalate cinesi

Tab. 3 - Report - Bianca - Mercato ingrosso

3 avocado

1 melanzana

3 zucchine grandi

4 grossi pezzi di carota

5 insalate iceberg

4 peperoni

$1.5 \mathrm{~kg}$ di pomodori ciliegia

5 limoni

5 pomodori insalata

6 cuori di bue

2 arance

$1 \mathrm{~kg}$ di peperoni italiani

3-4 kg di cetrioli

2 meloni

1 zucca

Pur essendo queste grandi quantità di cibo, soprattutto per quanto riguarda il mercato all'ingrosso, non rappresentano dei raccolti eccezionali. Al contrario, queste quantità indicano un raccolto usuale presso i differenti siti di riciclo. È facilmente comprensibile come la pulizia di tutti questi alimenti richieda una quantità considerevole di tempo. Durante la pulizia avviene una nuova selezione degli alimenti: si eliminano i pezzi corrotti e si asciugano accuratamente. 

mercato all'ingrosso supera notevolmente la quantità di cibo che un singolo individuo può consumare in un breve periodo di tempo (da 3 a 5 giorni) oltre il quale questi alimenti risulterebbero difficilmente edibili. Questo eccesso di riciclo, che possiamo chiamare "surplus di produzione", viene donato a conoscenti, consumato in banchetti collettivi e conviviali o, nel caso dei gruppi costituiti, distribuito attraverso mense sociali gratuite o a prezzo libero. Viene dunque inserito e circola nelle reti $\mathrm{d}$ i conoscenza dei recuperatori, alimentando così circuiti di reciprocità in cui lo scambio di cibo è costante e regolare. In questo modo, il surplus del reciclaje alimenta uno scambio materiale, un dono diffuso, che rafforza i legami tra i membri del gruppo di appartenenza di ogni recuperatore. La circolazione di questi beni descrive così uno scambio collettivo, continuo, materiale e, come vedremo, anche intellettuale, che si realizza all'interno di reti informali di conoscenza e condivisione in cui gli scambi non sono monetizzati ${ }^{11}$.

20 Coloro che beneficiano di questa circolazione sono persone vicine al reciclaje. Questa vicinanza non è determinata solo dalla partecipazione concreta alla pratica, ma anche dalla prossimità ideologica nei confronti del reciclaje. I recuperatori donano gli alimenti 
ad altri recuperatori ma anche a coloro che, pur non praticando il reciclaje, consumano alimenti donati e provenienti dall'attività di riciclo. Pertanto, la condivisione del cibo s'inserisce in reti esistenti, tra persone che condividono la stessa visione ideologica relativa al reciclaje, e ne rafforza i legami. Il reciclaje (e il flusso di beni che alimenta) non contribuisce alla creazione delle reti di reciprocità e condivisione in cui questi attori sono inseriti, ma costituisce piuttosto uno strumento che facilita il mantenimento o il consolidamento delle relazioni esistenti tra i recuperatori: esso delimita il gruppo e contribuisce alla sua esistenza.

Nessun riciclatore incontrato ha mai segnalato una relazione inaugurata da una donazione di cibo riciclato. È un'altra condizione che crea la relazione: la condivisione della socialità. Nessuno dei giovani riciclatori incontrati è originario della città di Granada $^{12}$. Le capacità relazionali di queste persone si rivelano dunque fondamentali per ricreare i legami "familiari" e amicali che l'emigrazione ha allentato. Lo scambio continuo di cibo fra i membri delle reti di conoscenza e condivisione non solo aumenta la varietà di beni che circolano, ma genera anche una reciprocità generalizzata che ricostituisce e riproduce una sorta di reciprocità familiare.

La distribuzione del cibo riciclato avviene sulla base di un principio di orizzontalità e reciprocità poiché è spesso uno scambio tra "pari". Questi scambi rivelano ciò che identifico come "strong ties" (Granovetter 1973) all'interno della rete del riciclatore. È lungo questi legami forti che possiamo osservare un'intensa circolazione basata sullo scambio reciproco e non monetizzato di beni materiali e immateriali. Lo scambio materiale relativo al reciclaje degli alimenti è difatti accompagnato da uno scambio immateriale e intellettuale all'interno delle reti dei riciclatori. Intendo dire che tra $\mathrm{i}$ riciclatori si verificano altri scambi (oltre al cibo). Le informazioni su nuovi luoghi "speciali" dove riciclare e nuove idee per la lavorazione e conservazione del cibo sono condivise, così come lo sono i contatti per lavori formali o informali, strumenti (borse, lampade, coltelli, frigo-congelatori o fornelli a gas), veicoli (biciclette, passeggini, auto o furgoni), ospitalità e, infine, molto tempo.

Così, i modi di conoscere e valutare la realtà, le conoscenze attinenti al reciclaje degli alimenti, come altri tipi di abilità e conoscenze in generale, circolano, vengono trasformati e sono riprodotti collettivamente all'interno della rete dei riciclatori. Pertanto, la rete diventa un mezzo di trasmissione di abilità, capacità, punti di forza e potenzialità trasferibili: essa permette alle persone di sostenersi a vicenda e persino di proteggersi l'un l'altro, socializzare e promuovere l'inclusione in un gruppo specifico (Roulleau-Berger 1999). Il dono, in questo caso, appare un elemento chiave e funzionale all'aiuto reciproco tra i membri appartenenti alla stessa rete. Grazie al ciclo completo del reciclaje (raccolta, trasformazione, distribuzione, consumo) le merci passano dallo stato di scoria del sistema di produzione (senza alcun valore d'uso e di scambio) a uno stato di bene con un valore di uso e di scambio, anche se non monetario. La trasfigurazione di questi beni in donazioni o in consumi collettivi mostra questo cambiamento di status. In tal modo, la circolazione o il consumo di questi prodotti rafforza i legami interpersonali tra pari esistenti all'interno delle reti di reciprocità.

\section{Lavoro, consumo e tempo libero}

24 Attraverso le interviste ho provato ad accedere alla visione di questi giovani riguardo al consumo e al lavoro e a confrontare i loro discorsi. Il mio obiettivo era, infatti, quello di 
rilevare e definire il livello d'inserimento di ogni recuperatore nel sistema di produzione dominante e la sua capacità di consumo.

Tutti i giovani recuperatori incontrati hanno - a differenza dei loro "omologhi" pensionati, persone senza fissa dimora, immigrati e gitani - un diploma di scuola secondaria. Spesso hanno una laurea triennale, in alcuni casi una laurea specialistica. La lunga scolarizzazione, combinata con una varietà di esperienze e implicazioni personali, contribuisce a far maturare una visione particolare della società. Questi giovani affermano che, attraverso la loro costruzione culturale individuale, hanno sviluppato una coscienza socio-politica che ha generato in loro un'avversione per il consumo smisurato della società in cui crescono e vivono.

Nei loro discorsi, i giovani recuperatori investono questa pratica di un carattere politico che giustifica l'azione. Spesso, i miei interlocutori hanno sottolineato che, a loro parere, la causa dei rifiuti depositati nei cassoni dell'immondizia e destinati al macero è un sistema produttivo totalmente concentrato su una produzione e su un consumo necessariamente in costante crescita; una società basata sull'accumulazione illimitata in cui il desiderio di consumare deriva meno dall'esistenza di un bisogno reale e sempre più dal desiderio di affermare uno status sociale, imitando il modello di coloro che si trovano appena a un gradino al di sopra (Latouche 2008). Essi descrivono dunque il reciclaje come una risposta al sistema dominante di produzione e consumo. I loro discorsi s'intersecano con la critica delle logiche lavorative dominanti e la scelta di un'auto-limitazione del consumo e del lavoro, che portano al rifiuto di bisogni percepiti come indotti.

L'avversione nei confronti di una società iperproduttivista e iperconsumista è identificata dai giovani riciclatori come il movente che li conduce a compiere una scelta, una volizione. In una società che istituzionalizza desideri e impulsi consumistici, questi giovani dichiarano di scegliere l'autolimitazione dei propri bisogni o, meglio, un'autolimitazione del consumo, un ridimensionamento dei bisogni (secondari), una repressione dei desideri indotti e una nozione alternativa di ricchezza.

Ma questa "disintossicazione" dall'iperconsumismo, questo rifiuto di ciò che Dominique Belpomme definisce "il cancro dell'umanità" (Belpomme 2007), è davvero volontario? Non del tutto. Questi giovani riciclatori, anche se hanno tutti almeno un titolo di studio superiore, vivono, come la maggior parte dei giovani spagnoli, in condizioni di lavoro caratterizzate dalla precarietà. Nei limiti e costrizioni che questa situazione induce, $i$ giovani recuperatori usano il reciclaje come uno strumento per ridurre la loro partecipazione al sistema di produzione. L'uscita (o l'entrata e l'uscita), volontaria o meno, dal mondo del lavoro rendono necessaria questa riduzione del consumo e allo stesso tempo la facilitano. Nel caso dei recuperatori da me intervistati, la riduzione del consumo supportata dal reciclaje rende possibile un'intermittenza del lavoro spesso rivendicata come volontaria e in qualche modo ricercata.

Questa rinuncia volontaria a un lavoro "normale", che per un giovane d'oggi in Spagna significa nella maggior parte dei casi lavoro precario, è favorita dall'ostilità nei confronti delle logiche lavorative prevalenti. Provoca fastidio la presenza e l'autorità di un "capo" e malessere la lunga durata dei tempi d'impiego che occupano gran parte della giornata lasciando spazi molto limitati d'autonomia a coloro che a tali tempi si sottomettono. Ciononostante, quasi tutti i recuperatori incontrati hanno un lavoro. Questo perché nella nostra società il soddisfacimento dei bisogni assoluti, cioè dei beni primari, è soggetto a scambio economico e domanda una partecipazione, per quanto 
parziale o puntuale, al sistema di produzione (Polanyi 2001). Benché il reciclaje garantisca (parzialmente o totalmente) il loro sostentamento alimentare, non lavorando i riciclatori non riuscirebbero a garantirsi i loro, pur bassi, livelli di consumo. Queste persone non possono ovviamente rinunciare ai bisogni essenziali ma non vogliono adeguarsi alle logiche lavorative dominanti. È per tale motivo che ricercano modalità di lavoro alternative per il raggiungimento di un reddito. Le loro traiettorie biografiche descrivono quindi percorsi sinuosi caratterizzati da spostamenti tra "attività a orientamento economico" (informali) e "attività economiche" (formali) (Cingolani 2005: 71). Attraverso l'accumulazione di esperienze e di attività varie, il comportamento di questi giovani rivela una «logica d'inserzione e, allo stesso tempo, di diversione dei vincoli del lavoro salariato» (ivi: 72). Le loro attività non richiedono lunghi orari di lavoro e garantiscono una quantità significativa di tempo libero.

Tra i lavori dei miei interlocutori vi sono: lo spaccio di droghe leggere, la vendita ambulante di manufatti di loro produzione, lavori agricoli stagionali, lavori saltuari a breve termine, giornalieri o part-time, spettacoli di strada. È il tempo libero, o liberato dal sistema salariale formale, che unisce chi pratica queste attività. In effetti, uno studente-lavoratore gestisce autonomamente la ripartizione delle ore di lavoro, lo studio e il suo tempo libero. Per uno spacciatore non v'è quasi alcuna differenza tra tempo libero e lavoro, inoltre il piccolo traffico di droga garantisce, nonostante il rischio, un buon rapporto tra tempo di lavoro e benefici. Allo stesso modo, un ambulante $\mathrm{o}$ un artista gestiscono autonomamente la propria attività di lavoro e tempo libero. In una città turistica come Granada, la vendita di prodotti artigianali e l'arte di strada, benché soggette alle condizioni meteorologiche e alle capacità individuali, garantiscono un buon rapporto tra tempo di lavoro e profitti generati. Un lavoratore a tempo parziale, anche se non può scegliere liberamente i suoi orari di lavoro, ha tuttavia molto tempo libero a sua disposizione. Ancor più, ovviamente, un disoccupato.

Quindi, i giovani recuperatori incontrati sul terreno riducono il consumo (secondario) sulla base di una scelta che è il risultato di una critica politico-sociale della società consumistica. Ciò non toglie che la soddisfazione dei bisogni primari (cibo, vestiti, casa, socialità) richieda una partecipazione, seppur parziale, contingente e saltuaria, al sistema di produzione. La partecipazione esclusivamente parziale e momentanea aumenta la quantità di tempo libero di questi individui. Questo tempo libero speso nella sfera della socialità non favorisce un'accumulazione di beni materiali ma permette di aumentare la ricchezza che deriva da beni relazionali: la convivialità, la conoscenza e il suo scambio reciproco (Latouche 2008). Inoltre, la condivisione nella sfera del gruppo sociale garantisce a questi individui la possibilità di acquisire una conoscenza versatile che permette loro di indirizzarsi verso settori economici coerenti con la loro filosofia di vita e di mettere le loro energie al servizio di attività che permettono di generare reddito ma che hanno caratteristiche diverse dalle logiche lavorative dominanti (Boni 2006).

32 Il tempo libero non deriva quindi dalla pratica del reciclaje, ma ne è un prerequisito. Prima di dedicarsi al reciclaje, questi giovani erano già in una situazione caratterizzata dalla discontinuità del lavoro. Ma così trasformato, il tempo libero non è più un tempo morto tra un lavoro e l'altro e non è più subordinato alla precarietà del sistema produttivo: diventa il tempo della costruzione e dello sviluppo individuale e collettivo (Cingolani 2005: 70-78). 
suggerito da Marshall Sahlins (1980), il termine "lavoro" indica le diverse modalità con le quali i gruppi umani modificano o utilizzano le risorse che il loro ambiente naturale offre per sopperire alle necessità dell'esistenza. Il reciclaje è, dunque, un lavoro poiché richiede una buona conoscenza del territorio (urbano, nel caso specifico) e l'impiego di competenze, energia e tempo. Quest'attività genera un reddito. Tale reddito non è monetario ma materiale, in questo caso cibo, e permette di allentare e ridurre la necessità di generare altro reddito (monetario) necessario per garantire le risorse, il cibo, indispensabile a questi individui. Il reciclaje è, quindi, uno strumento che permette di ridurre la sfera dei bisogni primari che possono essere soddisfatti solo attraverso uno scambio di mercato e attraverso il ricorso a relazioni monetizzate di scambio (su un mercato alimentare). Il reciclaje, infatti, riduce la quantità di cibo acquistata: si esce dall'acquisto riciclando ciò che il sistema scarta. Inoltre il tempo libero permette al reciclaje di incastrarsi nelle giornate di questi individui senza interferire con altre attività.

Ñuko, laureato in scienze economiche, dealer ${ }^{13}$ e riciclatore di lungo corso, mi spiega la sua visione:

Ñuko: Uomo, se hai un lavoro di 8 ore [... ] probabilmente non puoi perdere [tempo] [...] o sei molto stanco, capisci cosa dico? Anche questo succede alla gente [...] io non ho un lavoro, faccio quello che voglio [...] è un altro ritmo di vita... se tu hai un lavoro di 8 ore e hai soldi non vai al [mercato all'ingrosso] a metterti nei cassoni a raccogliere frutta, dopo lavarla, dopo metterti a cucinare [...] "pago 3 euro, pago 5 euro", cambia poco... la gente fa così. (Giugno 2009. Traduzione dell'autore)

\section{Conclusione}

All'inizio di quest'articolo ho provato a dare risalto al fatto che il prodotto del lavoro di riciclo è il risultato di una semplice, seppur accurata, ricerca nei cassonetti dei rifiuti urbani. L'accesso a tali cassonetti non è consentito a qualunque cittadino poiché la legislazione spagnola presenta disposizioni che normano e restringono tale accesso. Tuttavia, non solo la pratica del reciclaje è ampiamente tollerata da un punto di vista sociale ma oltre a ciò risulta palese la difficoltà per le forze dell'ordine di tenere sotto controllo i numerosi siti di riciclo disseminati sul terreno granadino ${ }^{14}$. Questa situazione garantisce ai riciclatori un libero accesso alle risorse di loro interesse. Inoltre i soli strumenti necessari alla pratica del reciclaje sono contenitori, facilmente recuperabili tra i rifiuti stessi, e, facoltativamente, l'uso di guanti. Il paragrafo "Lavoro, consumo e tempo libero" dimostra come la scelta dell'autolimitazione nella sfera del consumo comporti e renda necessario un tenore di vita relativamente basso. La diretta conseguenza, in questo caso, dell'autolimitazione del consumo è la riduzione della quantità di lavoro necessaria al raggiungimento della soddisfazione dei livelli di consumo dei riciclatori. Questa riduzione del lavoro rende necessaria, a sua volta, l'uscita da un consumo ordinario. Per questo motivo molti dichiarano nelle interviste di aver cominciato a praticare il reciclaje per necessità. Questa necessità, però, non sembra essere la conseguenza della contingenza economica spagnola sulle vite di queste persone, ma è una conseguenza indotta dalla scelta di autolimitazione dei consumi che si ripercuote sulla sfera lavorativa e sulla sua redditività di questi giovani recuperatori.

Inoltre è evidente che l'uscita, o l'entrare e uscire dal mercato del lavoro, lascia una grande quantità di tempo libero che viene utilizzato per indirizzare le energie verso attività che generano reddito materiale benché presentino caratteristiche diverse dalle 
logiche lavorative prevalenti (tra le altre, il reciclaje), per lo sviluppo della sfera della socialità, per l'accumulazione di beni relazionali e per la cura e la crescita individuale, ma anche per lo scambio. Uno scambio che è, come abbiamo visto, sia materiale sia intellettuale.

Sulla base della riflessione economica proposta da Sahlins potremmo quindi definire questo gruppo come opulento e convenire sul fatto che un'economia sostenibile esiste ed è possibile proprio qui nel cuore della "psuedo-opulenza" occidentale. Come spiega Serge Latouche:

Alcuni sono già riusciti individualmente a realizzare questa fuoriuscita dalla società [del lavoro], e queste esperienze possono indicare una strada, a patto di resistere all'ingranaggio dell'accumulazione illimitata e di difendersi dal ciclo infernale dei bisogni e del reddito. [...] Lavorare meno e diversamente può voler dire ritrovare il gusto del tempo libero, recuperare l'abbondanza perduta delle società dei cacciatori-raccoglitori descritta da Sahlins (Latouche 2008: 100).

L'uscita dalla società del lavoro richiede la riduzione del tempo di lavoro e il cambiamento del suo contenuto ma anche la riduzione e la "razionalizzazione" o, per meglio dire, il razionamento del consumo. Questi traguardi sono, però, il risultato di scelte ideologiche, fondate su una critica dell'attuale sistema, che richiedono impegno e la capacità di "mettersi in gioco". Questo percorso è faticoso e deve essere scelto consapevolmente poiché le forti pressioni della società vanno esattamente in senso opposto. Si tratta, per i miei interlocutori, di fare la scelta di vivere nel presente invece di sacrificare la vita presente al consumo e all'accumulazione di valori privi di valore (Brune 2005: 178). Sono scelte che indicano la volontà e la ricerca di una trasformazione sociale che deve necessariamente partire da ogni singolo individuo (Latouche 2008). L'individualismo, infatti, è diffuso tra i giovani riciclatori ma non è un freno a tale trasformazione.

Alla domanda «ti senti parte di un gruppo?», una giovane riciclatrice, laureanda in psicologia e buttadentro in un piccolo bar-ristorante del centro città, risponde:

Isabel: Mi sento parte di una rivoluzione... in realtà di una rivoluzione... non, non penso che ci sia un gruppo costituito né... né che abbia a che vedere qualcosa con un'altra persona che fa quello che fai tu... però sento che questa è una rivoluzione, lenta come tutte le rivoluzioni che non sono armate però sento che è una piccola rivoluzione (Aprile 2009. Traduzione dell'autore).

Eppure, per quanto questa sia una lenta rivoluzione contro la società consumistica, $\mathrm{i}$ riciclatori rimangono marginali al sistema capitalista e produttivista. Il reciclaje, infatti, nasce da un eccesso di tale sistema. Di tale eccesso il riciclatore si alimenta, si nutre, si sazia e alla fine non propone nessuna vera alternativa economica al nemico che si propone di abbattere.

\section{BIBLIOGRAFIA}

Barles S., 2005 L'invention des déchets urbains, France, 1790-1970, Champ Vallon, Seyssel. 
Bauman Z., 2009 Consumo, dunque sono, Laterza, Roma-Bari.

Bazin H., 2012 Etude qualitative portant sur les conditions de vie des biffins en Ile-de-France, Aurore, Paris.

Belpomme D., 2007 Avant qu'il ne soit trop tard, Fayard, Paris.

Black R., 2009 «Eating garbage: socially marginal food provisioning practices» in MacClancy J.et al. (eds), Consuming the inedible: neglected dimensions of food choice, Berghahn, New York: 141-149.

Boni S., 2006 Vivere senza padroni: antropologia della sovversione quotidiana, Eleuthera, Milano.

Bouillon F., 2009 Les mondes du squat, PUF, Paris.

Brune F., 2004 De l'ideologie, aujourd'hui, Paragon, Lyon.

Cingolani P., 2005 La précarité, Presses universitaires de France, Paris.

Counihan C., Siniscalchi V. (eds), 2014 Food Activism. Agency, Democracy and Economy, Bloomsbury Academic, London.

De Silguy C., 2009 Histoire des hommes et de leurs ordures, du Moyen Âge à nos jours, Le Cherche-Midi, Paris.

Granovetter M., 1973 «The Strength of Weak Ties» in American Journal of Sociology, Vol. 78, n. 6, The University of Chicago Press, Chicago: 1360-1380.

Hubert A., 1990 «Ethnologie et nutrition. L'alimentation comme pratique culturelle chez les Yao de Thaillande», in Fassin D. et Jaffré Y. (sous la direction de), Sociétés, développement et santé, Marketing/Ellipses, Paris: 259-272.

Kopytoff I., 1986 «The cultural biography of things: Commoditization as process», in Appadurai A. (ed.), The Social Life of Things, Cambridge University Press: 64-94.

Laffort B., 2008 Vous avez dit récupération? En enquête pour rendre compte des multiples formes de récupération dans le Nord de la France, USTL, Lille.

Latouche S., 2008 Breve trattato sulla decrescita serena, Bollati Boringhieri, Torino.

Mourad M., 2018 La lutte contre le gaspillage alimentaire en France et aux Etat-Unis, tesi inedita.

Orlando G., 2018 «Le reti alternative del cibo dopo la crisi. Teoria, ipotesi di lavoro e un caso studio esplorativo», Archivio Antropologico Mediterraneo [Online], Anno XXI, n. 20 (1).

Polanyi K., 2001 The great transformation: the political and economic origins of our time, Beacon Press, Boston.

Razetti N., 1891 Del furto campestre nella dottrina e nella giurisprudenza, Unione Tipografico-Editrice, Torino.

Roulleau-Berger L., 1999 Le travail en friche. Les mondes de la "petite" production urbaine, La Tour d'Aigues, L'Aube.

Sahlins M., 1980 L'economia dell'età della pietra: scarsità e abbondanza nelle società primitive, Bompiani, Milano.

Stuart T., 2009 Waste: uncovering the global food scandal, Penguin Books, London.

Varda A., 2000 Les glaneurs et la glaneuse, Ciné Tamaris, Paris.

Varda A., 2002 Deux Ans Après, C.N.D.P., Paris. 


\section{NOTE FINALI}

1. Letteralmente, riciclo.

2. Rispettivamente a Leonia (2011-12, 10 mesi) e Marsiglia (2014-17, 24 mesi) per i miei studi di $2^{\circ}$ e $3^{\circ}$ ciclo.

3. Svoltosi da gennaio a giugno 2009. Nel complesso ho condotto 45 interviste semi-strutturate e audio-registrate (con riciclatori, commercianti, agenti di sicurezza privata e autorità municipali) in lingua spagnola, inglese, francese e italiana. A queste ho affiancato numerose esperienze dirette di osservazione partecipante e la sistematica produzione di report di alimenti e rispettive quantità recuperati da ogni riciclatore incontrato. Per ragioni di spazio, nel presente articolo prenderò in esame i soli dati relativi ai giovani di età compresa tra i 20 e 35 anni.

4. Pratica antichissima, inscritta nei diritti e nelle pratiche dei beni comuni, menzionata esplicitamente anche nell'Antico Testamento (Razetti 1891: 22-33). Consiste nella ricerca e raccolta di spighe o di qualunque altro coltivo rimasto nei campi dopo la mietitura o la raccolta.

5. Durante il lungo lavoro di scrittura mi è stata posta spesso la domanda «perché Granada?». La mia risposta è sempre stata la stessa: «Perché Granada è la città più bella del mondo». Questo è probabilmente vero alla luce del grande numero di scrittori che le hanno dedicato pagine poetiche. Tuttavia, giustificare la scelta di un terreno di ricerca etnoantropologico attraverso dei parametri estetici non è sicuramente deontologicamente giustificabile. Cionondimeno, la relazione che s'instaura tra ricercatore e terreno è caratterizzata da una influenza reciproca: se il ricercatore sceglie, quando può, il terreno è perché il terreno, in precedenza, lo ha intrigato, lo ha sedotto. La scelta sarebbe potuta ricadere su una delle numerose città nelle quali pratiche di riciclo alimentare sono state già documentate e trattate: Lione e Torino (Black 2009), Londra (Stuart 2009), Lille (Laffort 2001), Parigi (Barles 2005; de Silguy 2009; Mourad 2018), Marsiglia (Bouillon 2009), per citarne solo alcune. Eppure, pragmaticamente, il terreno granadino è stato scelto sulla base di due elementi essenziali alla buona riuscita di una ricerca: la perfetta padronanza della lingua locale e la buona conoscenza della realtà sociale e urbana del terreno (Hubert 1990).

6. Deposito immondizia.

7. Baby-sitter serale.

8. Adotto questa classificazione in categorie (anziani e pensionati, persone senza fissa dimora, immigrati, gitani e giovani tra i 20 e i 35 anni) sulla base di informazioni raccolte attraverso brevi ma numerose interviste informali coi riciclatori. Questa categorizzazione ha come unico obiettivo di dare un rapido panorama della composizione generale dei recuperatori incontrati nei differenti punti d'osservazione poiché, nel quadro di una riflessione articolata, l'uso di queste stesse categorie impedisce un'analisi fine e precisa delle situazioni incontrate (es. giovani immigrati residenti in alloggi occupati, giovani gitani senza fissa dimora, anziani immigrati, ecc.). I numerosi casi di pluri-appartenenza e il problema della fluidità delle categorie mi hanno spinto nel corso della redazione della tesi di laurea ad abbandonarle in quanto strumento di analisi e a preferire un approccio basato sull'analisi delle competenze (competenze urbane, comunicativetransazionali e cognitive) mobilizzate dagli attori nella realizzazione della pratica di riciclo (Roulleau-Berger 1999, Bouillon 2009). Benché numerosi autori e studi usino le categorie sociali come strumento al servizio della comprensione scientifica, ritengo che generalmente tale pratica comporti una naturalizzazione dei fatti sociali equivocando le realtà osservate. In questi casi il rischio manifesto è quello di «semplificare e "oggettivizzare" gli individui, i quali vengono ridotti nella loro complessità sociale all'interno di categorie rigide». Come segnalato da Bazin (2012: 10), «possiamo categorizzare delle attività, dei comportamenti o delle posture, ma non l'umano nella sua essenza sociologica. Questo perché l'esistenza di una persona è una e non può essere ritagliata in sezioni attraverso un discorso erudito».

9. Tutte le interviste presenti in quest'articolo sono state raccolte in lingua spagnola. 
10. Durante la ricerca ho eseguito 112 report, redatti personalmente e nelle immediate vicinanze del sito di recupero. La produzione e raccolta di questi (e altri) dati non è mai stata delegata ai miei interlocutori per garantire la massima veridicità dei dati raccolti.

11. Nel caso delle mense a prezzo libero, lo scambio è nella maggior parte dei casi monetizzato. Tuttavia, la libertà individuale nella valutazione del prezzo da corrispondere mostra che l'intero sistema di definizione del valore (monetario o no) dell'alimento riciclato è diverso da quello dominante del calcolo del profitto in un contesto di mercato. Ciò a conferma che, come segnalato da Orlando (2018), le norme che permettono la determinazione del valore di un oggetto-merce «sono intrinsecamente sociali [poiché] il valore economico è un valore culturale, dopotutto».

12. Provenienti da altre città spagnole, e da Francia, Italia, Germania, Irlanda, Polonia, Belgio, Repubblica Ceca, Cile e Brasile.

13. Persona dedita al piccolo spaccio, nel caso specifico, di droghe leggere.

14. Inoltre in un paio di occasioni ho avuto modo di osservare riciclatori all'opera accanto alle forze di polizia.

\section{RIASSUNTI}

L'articolo analizza le pratiche informali di riciclo alimentare: la ricerca, la valorizzazione, la circolazione e il consumo di cibo scartato dalla catena alimentare urbana e la trasformazione di tale rifiuto in un oggetto commestibile e familiare. Ogni giorno, i riciclatori "informali" di alimenti si muovono nello spazio urbano e lo praticano in base ai vincoli e alle opportunità che la città offre loro. L'osservazione rigorosa delle loro azioni rivela che essi sviluppano conoscenze specifiche e funzionali alla pratica del riciclo alimentare. Le abilità sono mobilitate per decodificare ed esplorare la città e le sue attività; per interagire con attori e norme; per recuperare e trasformare gli alimenti utilizzati non solo per l'alimentazione ma anche come risorsa sociale per creare e consolidare gruppi attorno alla condivisione del cibo. In questo modo momenti e spazi di sopravvivenza diventano anche momenti e spazi d'innovazione dove le abilità, le competenze e le conoscenze relative al riciclo degli alimenti circolano, vengono trasformate e riprodotte collettivamente all'interno di questi gruppi. Pertanto, la rete diventa un mezzo di trasmissione di abilità, capacità, punti di forza e potenzialità trasferibili: essa permette alle persone di sostenersi a vicenda e persino di proteggersi l'un l'altra, socializzare e promuovere l'inclusione in un gruppo specifico. In questo contesto, le pratiche di riciclo alimentare appaiono come tattiche quotidiane volte al sostentamento di individui e gruppi di "attivisti", in cui la produzione e riproduzione di reti di solidarietà e condivisione definiscono economie morali parallele all'economia del mercato.

This paper analyses informal food-recycling practices: the research, reclamation, circulation, and consumption of food rejected from the urban food cycle and the transformation of garbage into an edible and familiar object. Every day, informal food-recyclers play with the urban space according to the constraints and opportunities that the city offers them. Rigorous observation of their practices reveals that they develop specific knowledge that is functional to the practice of salvaging food. Skills are mobilized to decode and explore the city and its activities; to interact with actors and norms; to reclaim and transform food used not only for nutrition but also as a social resource for creating and consolidating groups around food-sharing. So, moments and spaces of survival become also moments and spaces for innovation where skills, abilities and knowledge related to food-recycling circulate, are transformed and reproduced collectively within these groups. Thus, the informal network becomes the means of transmission of transferable skills, strengths and potentials and provides to individuals a support and even protects, socializes and promotes inclusion in a specific group. In this context, food-recycling 
practices appear as daily tactical practices, aimed at maintaining individual and "activist" groups, in which the production and reproduction of solidarity and sharing networks define moral economies parallel to market's economy.

INDICE

Keywords : food-recycling, waste, work, consumption, free time

Parole chiave : riciclo alimentare, rifiuti, lavoro, consumo, tempo libero

\section{AUTORE}

\section{GIORGIO CASSONE}

Centre Norbert Elias, EHESS giorgio.cassone@ehess.fr 